\title{
Adaptive Channel Estimation Based on an Improved Norm-Constrained Set-Membership Normalized Least Mean Square Algorithm
}

\author{
Yingsong Li, Zhan Jin, and Yanyan Wang \\ College of Information and Communications Engineering, Harbin Engineering University, Harbin 150001, China \\ Correspondence should be addressed to Yingsong Li; liyingsong@ieee.org
}

Received 1 August 2016; Revised 7 October 2016; Accepted 12 October 2016; Published 11 January 2017

Academic Editor: Nathalie Mitton

Copyright (c) 2017 Yingsong Li et al. This is an open access article distributed under the Creative Commons Attribution License, which permits unrestricted use, distribution, and reproduction in any medium, provided the original work is properly cited.

\begin{abstract}
An improved norm-constrained set-membership normalized least mean square (INCSM-NLMS) algorithm is proposed for adaptive sparse channel estimation (ASCE). The proposed INCSM-NLMS algorithm is implemented by incorporating an $l_{p}$-norm penalty into the cost function of the traditional set-membership normalized least mean square (SM-NLMS) algorithm, which is also denoted as $l_{p}$-norm penalized SM-NLMS (LPSM-NLMS) algorithm. The derivation of the proposed LPSM-NLMS algorithm is given theoretically, resulting in a zero attractor in its iteration. By using this proposed zero attractor, the convergence speed is effectively accelerated and the channel estimation steady-state error is also observably reduced in comparison with the existing popular SM-NLMS algorithms for estimating exact sparse multipath channels. The estimation behaviors are investigated via a typical sparse wireless multipath channel, a typical network echo channel, and an acoustic channel. The computer simulation results show that the proposed LPSM-NLMS algorithm is better than those corresponding sparse SM-NLMS and traditional SM-NLMS algorithms when the channels are exactly sparse.
\end{abstract}

\section{Introduction}

Set-membership filtering (SMF) has been extensively studied for adjusting the filter coefficients whose output error is limited by a prior bound [1-3]. Based on the SMF technique, a normalized least mean square (NLMS) algorithm with SMF has been proposed to reduce the data updates and iterations for getting the same or lower steady-state error floor, which is denoted as set-membership NLMS (SMNLMS) algorithm [1]. Since the prior bound is important for SM-NLMS algorithm, error-bound development methods have been proposed such as parameter-dependent technique [4-6]. In recent years, the SM-NLMS algorithm has been widely used for channel estimation, adaptive control, echo cancellation, and system identification [3]. Although the SMNLMS algorithm can provide excellent estimation performance, its behavior may be degraded for estimating sparse system such as sparse multipath wireless communication channel.
As we know, broadband techniques have been becoming an amazing choice for next-generation mobile communications [7-9]. For example, the 5G communication system occupies a wide bandwidth. Additionally, the coherent detection needs accurate channel state information (CSI) at the receiver side [7]. Moreover, the CSI is always obtained by using adaptive channel estimations (ACE), which is realized by using least mean square (LMS), NLMS, and SM-NLMS algorithms. On the other hand, the measurement of broadband multipath channel given by Vuokko et al. shows that the broadband channel has few dominated channel coefficients whose magnitudes are nonzeros, while most of the channel taps are zeros [10]. Such broadband channel is called sparse channel. Recently, sparse channel estimation has been widely studied to exploit its in-nature sparse structure, which is implemented based on the compressed sensing (CS) [11-15] and sparse adaptive filtering (SAF) algorithms [16-23]. Both of these algorithms can achieve good channel estimation. However, the CS-based broadband channel estimation is 
more complex than the SAF algorithms because the CS-based channel estimations require measurement matrix construction which is bounded by restricted isometry property [24].

SAF algorithms are usually implemented on the basis of proportionate $[25,26]$ and zero-attracting (ZA) techniques [16-23]. In the frame of proportionate technique, a typical algorithm is proportionate NLMS (PNLMS) which needs to assign an independent step size to each channel coefficient based on the latest estimation [25]. As a result, it results in the fact that the large step size is exerted on the large coefficients. However, it can improve the channel estimation performance at initial stage; its convergence slows down quickly and is even worse than the traditional NLMS algorithm. The zeroattracting technique has been proposed and introduced to exploit the sparse least mean square (LMS) algorithm which is realized by exerting an $l_{1}$-norm penalty on the channel vector and cooperating it into the cost function of the traditional LMS algorithm [16]. The principle of the zero-attracting LMS algorithms is to use various norm penalties in their cost functions to form desired zero attractors which attract the small channel coefficients to zero quickly. According to the excellent performance, the ZA technique has been expanded to the NLMS [7], affine projection algorithm [27$30]$, least mean fourth [31, 32], and other ZA adaptive filtering algorithms [33, 34]. Among these ZA adaptive filtering (AF) algorithms, sparse NLMS algorithm has been widely studied and applied for broadband channel estimation [7]. Recently, the ZA technique has been used for exploiting sparse SMNLMS in channel estimation applications for achieving low complexity, including ZA SM-NLMS (ZASM-NLMS) and reweighted ZASM-NLMS (RZASM-NLMS) algorithms [23].

In this paper, an improved norm-constrained setmembership normalized least mean square (INCSM-NLMS) algorithm is proposed for adaptive sparse channel estimation (ASCE), which is implemented by modifying the cost function of traditional SM-NLMS algorithm via adding a $l_{p^{-}}$ norm penalty term and is also denoted as $l_{p}$-norm penalized SM-NLMS (LPSM-NLMS) algorithm. The derivation of the proposed LPSM-NLMS algorithm is given in detail. Our simulation results obtained from sparse channel estimations show that the proposed LPSM-NLMS algorithm outperforms the standard SM-NLMS, ZASM-NLMS and RZASM-NLMS algorithms with respect to the channel estimation behaviors.

The rest of this paper is constructed as follows. Section 2 reviews the SAF theory and the standard SM-NLMS algorithm for channel estimation. Section 3 gives the proposed LPSM-NLMS algorithm and its derivation in detail. Computer simulation results are given in Section 4 in comparison with the estimation behaviors of standard SM-NLMS, ZASMNLMS, and RZASM-NLMS algorithms. Section 5 concludes this paper.

\section{SAF Theory and SM-NLMS Algorithm}

2.1. SAF Theory. Consider an input training signal $\mathbf{x}(n)=$ $[x(n), x(n-1), \ldots, x(n-N-1)]^{T}$, an additive white Gaussian noise (AWGN) signal $v(n)$, and a received signal $d(n)$ to illustrate a classical adaptive channel estimation. The input signal $\mathbf{x}(n)$ is transmitted to an unknown FIR channel and the output of the channel denotes as $y(n)$ which is obtained by $y(n)=\mathbf{x}^{T}(n) \mathbf{w}$. At the receiver side, the received signal $d(n)$ is gotten with $v(n)$. The adaptive filtering is to mimic the instantaneous estimation error $e(n)$ which is defined as the difference between the received signal and estimated output. Thus, we have $e(n)=d(n)-\mathbf{x}^{T}(n) \widehat{\mathbf{w}}(n)$, where $\widehat{\mathbf{w}}(n)$ denotes as the estimated output vector.

As we know, the classical adaptive filter algorithms are used to estimate the unknown channel $\mathbf{w}$ through minimizing an error function which has a relationship with the instantaneous error $e(n)[3,35,36]$. For example, the LMS algorithm uses $e^{2}(n)$. Also, the NLMS algorithm introduces a normalized power of the input signal to improve the performance of the LMS algorithms. Recently, a special bound has been used on the magnitude of $e(n)$, such as SAF scheme $[1,2]$. The SAF algorithms use an interested subspace to model $e(n)$. We give a model space $S$, which is comprised of the input vector-desired output pairs (IVDOPs). As for the SAF theory, an error criterion has been utilized to bound $S$. Parameter estimation is bounded by a specified parameter $\gamma$ for all the data obtained from $S$. Therefore, SMF algorithm is to choose a special set in the parameter space which is different from the point estimation. The illustration of the SMF principle is expressed as

$$
|e(n)|^{2} \leq \gamma^{2} \quad \forall(\mathbf{x}(n), d(n)) \in S,
$$

where $(\mathbf{x}(n), d(n))$ is the IVDOP. As for any combination of $\forall(\mathbf{x}(n), d(n)) \in S$, we can get the solution of possible vectors $\mathbf{w}$ by using the following formula [1-3]:

$$
\Theta=\bigcap_{(\mathbf{x}(n), d(n)) \in S}\left\{\widehat{\mathbf{w}} \in \mathbb{R}^{N}:\left|d(n)-\mathbf{x}^{T}(n) \widehat{\mathbf{w}}(n)\right| \leq \gamma\right\},
$$

where $\mathbb{R}^{N}$ is a vector space whose dimension is $N$. If $k$ IVDOPs $\left\{x_{i}(n), d_{i}(n)\right\}_{i=1}^{k}$ are utilized to train the filter, the measurement set for the SAF algorithms is written as $[1,2]$

$$
H_{k}=\left\{\widehat{\mathbf{w}} \in \mathbb{R}^{N}:\left|d_{k}(n)-\mathbf{x}_{k}^{T}(n) \widehat{\mathbf{w}}_{k}(n)\right| \leq \gamma\right\} .
$$

The SAF algorithms can find out the solutions that belong to an exact set $\Psi_{k}$ which contains $k$ observed IVDOPs:

$$
\Psi_{k}=\bigcap_{i=1}^{k} H_{i}
$$

Actually, the set $\Theta$ is a subset of the exact membership set $\Psi_{k}$ in each iteration.

2.2. SM-NLMS Algorithm. The SAF technique is integrated into the NLMS algorithm to get the traditional setmembership NLMS (SM-NLMS) algorithm. From our previous knowledge of SAF algorithms, the updated equation of the traditional SM-NLMS algorithm [1-3] is similar to that of the classical NLMS algorithm [3, 35, 36]. However, the principle of the SM-NLMS algorithm is different from the classical NLMS algorithm. The SM-NLMS algorithm minimizes $\|\widehat{\mathbf{w}}(n+1)-\widehat{\mathbf{w}}(n)\|^{2}$ under a special constraint of $\widehat{\mathbf{w}}(n+1) \in H_{k}$. For any $\widehat{\mathbf{w}}(n) \notin H_{k}$, we use the following 
optimization problem to get the solution of the SM-NLMS algorithm, which is expressed as [1-3]

$$
\begin{array}{cl}
\operatorname{minimize} & \|\widehat{\mathbf{w}}(n+1)-\widehat{\mathbf{w}}(n)\|^{2} \\
\text { subject to: } & d(n)-\mathbf{x}^{T}(n) \widehat{\mathbf{w}}(n+1)=\gamma .
\end{array}
$$

To find out the solution of (5), Lagrange multiplier method is employed to calculate its minimization. As a result, the cost function of the SM-NLMS algorithm can be written as

$$
\begin{aligned}
J_{\mathrm{SM}}(n)= & \|\widehat{\mathbf{w}}(n+1)-\widehat{\mathbf{w}}(n)\|^{2} \\
& +\lambda\left(d(n)-\mathbf{x}^{T}(n) \widehat{\mathbf{w}}(n+1)-\gamma\right) .
\end{aligned}
$$

We can get the update equation of the SM-NLMS algorithm written as

$$
\begin{aligned}
& \widehat{\mathbf{w}}(n+1) \\
& = \begin{cases}\widehat{\mathbf{w}}(n)+\frac{\mathbf{x}(n)}{\mathbf{x}^{T}(n) \mathbf{x}(n)+\delta}(e(n)-\gamma) & \text { if }|e(n)|>\gamma \\
\widehat{\mathbf{w}}(n) & \text { otherwise, }\end{cases}
\end{aligned}
$$

which is also given by

$$
\widehat{\mathbf{w}}(n+1)=\widehat{\mathbf{w}}(n)+\frac{\mathbf{x}(n)}{\mathbf{x}^{T}(n) \mathbf{x}(n)+\delta} \alpha e(n),
$$

where

$$
\alpha= \begin{cases}1-\frac{\gamma}{|e(n)|} & \text { if }|e(n)|>\gamma \\ 0 & \text { otherwise }\end{cases}
$$

Here, parameter $\delta>0$ is a regulation parameter which is always very small and is to avoid dividing by zero. From (9), we can see that the SM-NLMS algorithm improves the performance of the classical NLMS algorithm by introducing an adaption parameter $\alpha[1-3]$.

\section{Proposed LPSM-NLMS Algorithms}

Although zero-attracting SM-NLMS (ZASM-NLMS) and reweighting ZASM-NLMS (RZASM-NLMS) algorithms have been proposed and they can well estimate the sparse channels, the sparsity of the multipath channel needs to be exploited further. In this section, we derive our proposed LPSM-NLMS algorithm based on the CS concepts reported in $[36,37]$. The proposed LPSM-NLMS algorithm is carried out by incorporating a $l_{p}$-norm [18] constrained channel coefficient vector into the cost function of the traditional SM-NLMS algorithm. Then, we can get the new cost function of our proposed LPSM-NLMS algorithm for $\widehat{\mathbf{w}}(n+1) \notin H_{k}$, which is to implement the following minimization optimization problem [23]:

$$
\begin{array}{cl}
\operatorname{minimize} & \|\widehat{\mathbf{w}}(n+1)-\widehat{\mathbf{w}}(n)\|^{2}+\gamma_{p}\|\widehat{\mathbf{w}}(n+1)\|_{p} \\
\text { subject to: } & d(n)-\mathbf{x}^{T}(n) \widehat{\mathbf{w}}(n+1)=\gamma_{\mathrm{LP}},
\end{array}
$$

where $\gamma_{p}$ is a zero-attracting strength controlling factor. Similar to the ZASM-NLMS and RZASM-NLMS algorithms, the Lagrange multiplier method is employed to find out the solution of the above optimization problem. Then, the cost function of the proposed LPSM-NLMS algorithm is given by

$$
\begin{aligned}
J(n)= & \|\widehat{\mathbf{w}}(n+1)-\widehat{\mathbf{w}}(n)\|^{2} \\
& +\lambda\left(d(n)-\mathbf{x}^{T}(n) \widehat{\mathbf{w}}(n+1)-\gamma_{\mathrm{LP}}\right) \\
& +\gamma_{p}\|\widehat{\mathbf{w}}(n+1)\|_{p} .
\end{aligned}
$$

On the basis of the Lagrange multiplier method, we let

$$
\begin{aligned}
\frac{\partial J(n)}{\partial \widehat{\mathbf{w}}(n+1)} & =0, \\
\frac{\partial J(n)}{\partial \lambda} & =0 .
\end{aligned}
$$

Then, we can get

$$
\begin{aligned}
& 2[\widehat{\mathbf{w}}(n+1)-\widehat{\mathbf{w}}(n)]-\mathbf{x}(n) \\
& \quad+\gamma_{p} \frac{\left(\|\widehat{\mathbf{w}}(n)\|_{p}\right)^{(1-p)} \operatorname{sgn}(\widehat{\mathbf{w}}(n))}{|\widehat{\mathbf{w}}(n)|^{(1-p)}}=0, \\
& d(n)-\mathbf{x}^{T}(n) \widehat{\mathbf{w}}(n+1)-\gamma_{\mathrm{LP}}=0 .
\end{aligned}
$$

By taking (13) and (14) into consideration, we can get

$$
\begin{aligned}
\frac{\lambda}{2}=\left[\mathbf{x}^{T}(n) \mathbf{x}(n)\right]^{-1}\left\{e(n)-\gamma_{\mathrm{LP}}\right\} \\
+\frac{\gamma_{p}}{2}\left[\mathbf{x}^{T}(n) \mathbf{x}(n)\right]^{-1} \mathbf{x}^{T}(n) \\
+\frac{\left(\|\widehat{\mathbf{w}}(n)\|_{p}\right)^{(1-p)} \operatorname{sgn}(\widehat{\mathbf{w}}(n))}{|\widehat{\mathbf{w}}(n)|^{(1-p)}} .
\end{aligned}
$$

Combining (13) and (15), we obtain

$$
\begin{aligned}
\widehat{\mathbf{w}}(n+1) & \\
= & \widehat{\mathbf{w}}(n)+\frac{\mathbf{x}(n)}{\mathbf{x}^{T}(n) \mathbf{x}(n)}\left\{e(n)-\gamma_{\mathrm{LP}}\right\} \\
& +\frac{\gamma_{p}}{2}(\mathbf{B}(n)-\mathbf{I}) \frac{\left(\|\widehat{\mathbf{w}}(n)\|_{p}\right)^{(1-p)} \operatorname{sgn}(\widehat{\mathbf{w}}(n))}{|\widehat{\mathbf{w}}(n)|^{(1-p)}},
\end{aligned}
$$

where $\mathbf{B}(n)=\left\{\mathbf{x}(n) \mathbf{x}^{T}(n) / \mathbf{x}^{T}(n) \mathbf{x}(n)-\mathbf{I}\right\}$. From the cost function of the proposed LPSM-NLMS algorithm, we know that the difference between the SM-NLMS and LPSM-NLMS algorithms is the minimization of $\gamma_{p}\|\widehat{\mathbf{w}}(n+1)\|_{p}$. Thus, we can simplify the optimization of the LPSM-NLMS to be

$$
\begin{aligned}
\operatorname{minimize} & \frac{1}{2}\|\widehat{\mathbf{w}}(n+1)-\widehat{\mathbf{w}}(n)\|^{2} \\
& +\frac{1}{2}\left\|e(n)-\gamma_{\mathrm{LP}}\right\|_{\mathbf{S}(n)}^{2}+\gamma_{p}\|\widehat{\mathbf{w}}(n+1)\|_{p},
\end{aligned}
$$


where $\mathbf{S}(n)=\left[\mathbf{x}^{T}(n) \mathbf{x}(n)\right]^{-1} \cdot\left\|e(n)-\gamma_{\mathrm{LP}}\right\|_{\mathbf{S}(n)}^{2}$ is defined as $\left\|e(n)-\gamma_{\mathrm{LP}}\right\|_{\mathbf{S}(n)}^{2}=\left[e(n)-\gamma_{\mathrm{LP}}\right]^{T} \mathbf{S}(n)\left[e(n)-\gamma_{\mathrm{LP}}\right]$. By using the Lagrange multiplier method on (17), we can get the updated equation of the LPSM-NLMS algorithm, which is given by

$$
\begin{aligned}
\widehat{\mathbf{w}}(n+1)= & \widehat{\mathbf{w}}(n)+\frac{\mathbf{x}(n)}{\mathbf{x}^{T}(n) \mathbf{x}(n)+\delta} \alpha e(n) \\
& -\rho \frac{\left(\|\widehat{\mathbf{w}}(n)\|_{p}\right)^{(1-p)} \operatorname{sgn}(\widehat{\mathbf{w}}(n))}{|\widehat{\mathbf{w}}(n)|^{(1-p)}},
\end{aligned}
$$

where $\alpha$ is the same as (9). Here, a zero-attracting factor $\rho>0$ is introduced to give a balance between the channel estimation behavior and the penalty strength of $\widehat{\mathbf{w}}(n)$. Here, $\operatorname{sgn}[\cdot]$ is defined as

$$
\operatorname{sgn}[w]= \begin{cases}\frac{w}{|w|}, & w \neq 0 \\ 0, & w=0 .\end{cases}
$$

In order to avoid dividing by zero, (18) is rewritten as

$$
\begin{aligned}
\widehat{\mathbf{w}}(n+1)= & \widehat{\mathbf{w}}(n)+\frac{\mathbf{x}(n)}{\mathbf{x}^{T}(n) \mathbf{x}(n)+\delta} \alpha e(n) \\
& -\rho \frac{\left(\|\widehat{\mathbf{w}}(n)\|_{p}\right)^{(1-p)} \operatorname{sgn}(\widehat{\mathbf{w}}(n))}{|\widehat{\mathbf{w}}(n)|^{(1-p)}+\varepsilon} .
\end{aligned}
$$

It is worth noting that the proposed LPSMNLMS algorithm introduces an additional term $-\rho\left(\left(\|\widehat{\mathbf{w}}(n)\|_{p}\right)^{(1-p)} \operatorname{sgn}(\widehat{\mathbf{w}}(n)) /\left(|\widehat{\mathbf{w}}(n)|^{(1-p)}+\varepsilon\right)\right)$ which is used for enforcing the filter coefficients to zero quickly. In LPSMNLMS algorithm, we can control the zero-attracting strength by adjusting the parameter $\rho$.

\section{Results and Discussion}

In order to verify the performance of the proposed LPSMNLMS algorithm, we employ a sparse channel and a network echo channel to analyze our proposed LPSM-NLMS algorithm. Also, its performance is compared with NLMS, SM-NLMS, ZA-NLMS, ZASM-NLMS, and RZASM-NLMS algorithms with respect to steady-state behavior. Monte Carlo simulation with 100 runs is used for obtaining each simulation point.

In the first investigation, a sparse multipath channel is used for assessing the estimation behavior of the proposed LPSM-NLMS algorithm. Here, the sparse channel has 16 taps with $K$ dominant ones, which is similar to [7, 16-23, 26-34, 38]. The $K$ dominant channel taps are gotten from a Gaussian distribution, which is subjected to $\|\widehat{\mathbf{w}}\|_{2}^{2}=1$. Moreover, the $K$ dominant channel taps are randomly distributed within the length of the sparse channel. The input training signal $\widehat{\mathbf{x}}(n)$ is a white Gaussian random signal. The channel output is in the presence of an AWGN $v(n)$ which is assumed to be independent with $\widehat{\mathbf{x}}(n)$. The channel estimation performance is investigated under signal-to-noise ratio (SNR) of $10 \mathrm{~dB}$, $20 \mathrm{~dB}$, and $30 \mathrm{~dB}$. The channel estimation performance is evaluated by mean square error (MSE) which is defined as
MSE $=E\|\mathbf{w}-\widehat{\mathbf{w}}(n)\|^{2}$. In this simulation, the simulation parameters optimized to obtain the same convergence rate at the initial stage are $\mu_{\mathrm{NLMS}}=\mu_{\mathrm{ZA}-\mathrm{NLMS}}=0.5, \gamma_{\mathrm{ZA}}=\gamma_{\mathrm{RZA}}=$ $\gamma_{\mathrm{LP}}=\gamma=0.707 \delta_{v}^{2}, \rho_{\mathrm{ZA}-\mathrm{NLMS}}=\rho_{\mathrm{ZA}}=\rho_{\mathrm{RZA}}=5 \times 10^{-4}$, $\rho=6 \times 10^{-5}, p=0.5$, and $\varepsilon=0.001$, where $\mu_{\mathrm{NLMS}}$ and $\mu_{\text {ZA-NLMS }}$ are the step sizes of NLMS and ZA-NLMS algorithms, respectively. $\rho_{\mathrm{ZA}-\mathrm{NLMS}}, \rho_{\mathrm{ZA}}$, and $\rho_{\mathrm{RZA}}$ are the zero attraction factors for ZA-NLMS, ZASM-NLMS, and RZASM-NLMS algorithms, respectively. The channel estimation behaviors with $K=1$ and $K=2$ are shown in Figures 1, 2, and 3 for SNR $=10 \mathrm{~dB}, \mathrm{SNR}=20 \mathrm{~dB}$, and SNR $=30 \mathrm{~dB}$, respectively. We can see that our proposed LP-NLMS algorithm achieves fast convergence speed and smallest MSE. When $K$ increases from 1 to 2, the MSE has slightly deteriorated, which is caused by the reduced sparsity. It is worth noting that the proposed LPSM-NLMS algorithm can obtain more gains when SNR ranges from $10 \mathrm{~dB}$ to $30 \mathrm{~dB}$.

Next, we verify our proposed LPSM-NLMS algorithm over a typical network echo channel. An example of a typical network echo channel is shown in Figure 4, where the channel has a length of 256 and it has 16 dominant taps. In this experiment, we use $\zeta_{12}(\mathbf{w})=(\mathrm{N} /(\mathrm{N}-$ $\sqrt{N}))\left(1-\|\mathbf{w}\|_{1} / \sqrt{N}\|\mathbf{w}\|_{2}\right)[38,39]$ to define the sparseness measure of the network echo channel. Two sparse levels with $\zeta_{12}(\mathbf{w})=0.8222$ and $\zeta_{12}(\mathbf{w})=0.7362$ are utilized to assess the estimation performance of the proposed LPSMNLMS algorithm. To get the same convergence speed rate, the simulation parameters are $\rho_{\mathrm{ZA}-\mathrm{NLMS}}=5 \times 10^{-6}, \rho_{\mathrm{ZA}}=$ $\rho_{\text {RZA }}=6 \times 10^{-6}$, and $\rho=8 \times 10^{-6}$. Figure 5 illustrates the tracking behavior and the channel estimation performance of the proposed LPSM-NLMS algorithm, which is driven by a white input signal. It is found that the proposed LPSMNLMS algorithm outperforms the traditional NLMS, SMNLMS, ZA-NLMS, ZASM-NLMS, and RZASM-NLMS with respect to the convergence speed and the MSE. Even though the sparseness measure is reduced from $\zeta_{12}(\mathbf{w})=0.8222$ to $\zeta_{12}(\mathbf{w})=0.7362$, our proposed LPSM-NLMS algorithm is still superior to the existing sparse SM-NLMS algorithms.

Finally, an acoustic impulse response (AIR) which is obtained by using the image method in $[40,41]$ is employed to further investigate the performance of the proposed LPSM-NLMS algorithm. Here, an example of AIR with a length $N=512$ is utilized to give a verification of the proposed LPSM-NLMS algorithm and the channel is obtained in a room of dimension $10 \times 10 \times 6$ at a sample frequency of $16 \mathrm{kHz}$. Figure $6(\mathrm{a})$ gives the normalized AIR channel (the MATLAB code for producing this channel can be found at http://www.commsp.ee.ic.ac.uk/ pl103/research $. h t m l)$. Moreover, loudspeaker-microphone distance is $2 \mathrm{~m}$ in a loudspeaker-room-microphone system (LRMS) whose reflection coefficient is set to 0.3 . In this simulation, the parameters are $\rho_{\mathrm{ZA}-\mathrm{NLMS}}=\rho_{\mathrm{ZA}}=\rho_{\mathrm{RZA}}=5 \times 10^{-6}, \rho=$ $1 \times 10^{-8}$. We can see that our proposed LPSM-NLMS is still superior to the NLMS, SM-NLMS, ZA-NLMS, ZASMNLMS, and RZASM-NLMS algorithms. Additionally, the proposed LPSM-NLMS converges faster than the traditional SM-NLMS algorithm and it can also achieve some gain in comparison with the previously presented RZASM-NLMS 


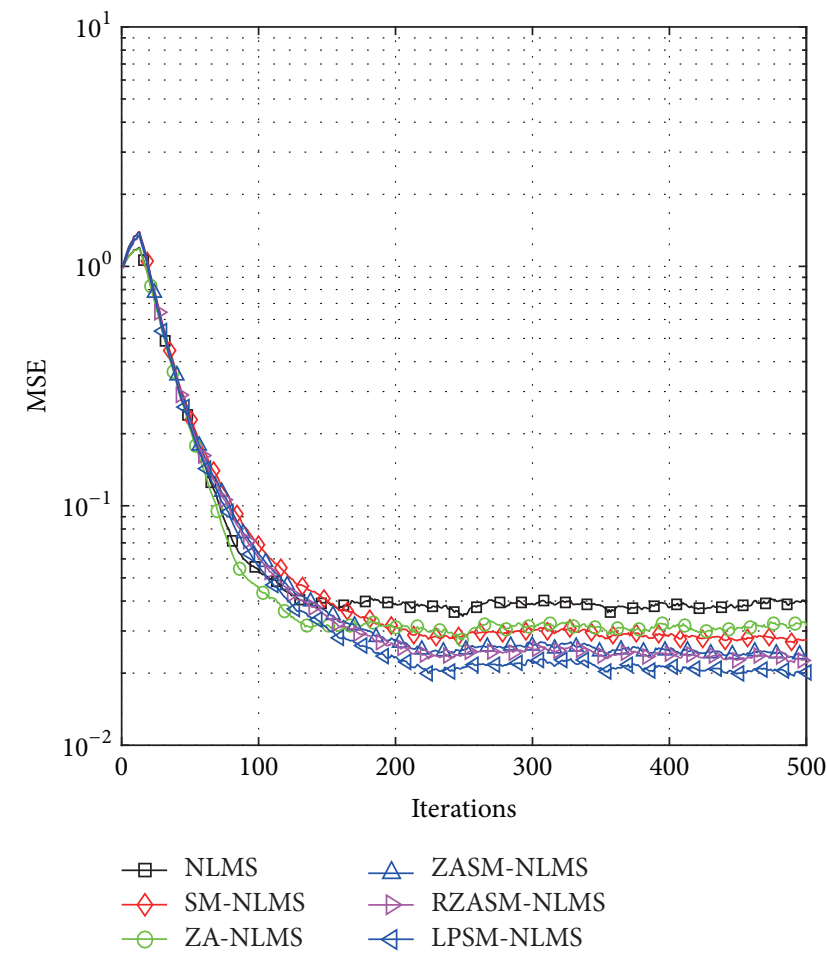

(a) $K=1$

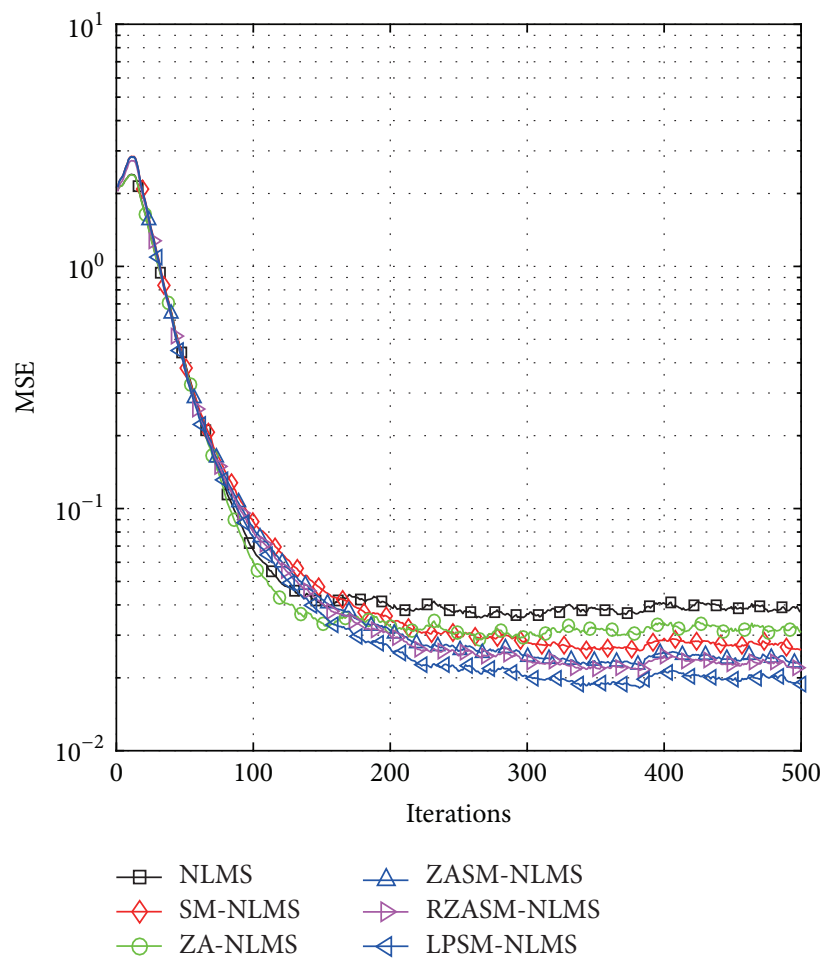

(b) $K=2$

FIGURE 1: Sparse channel estimation behavior for SNR $=10 \mathrm{~dB}$.

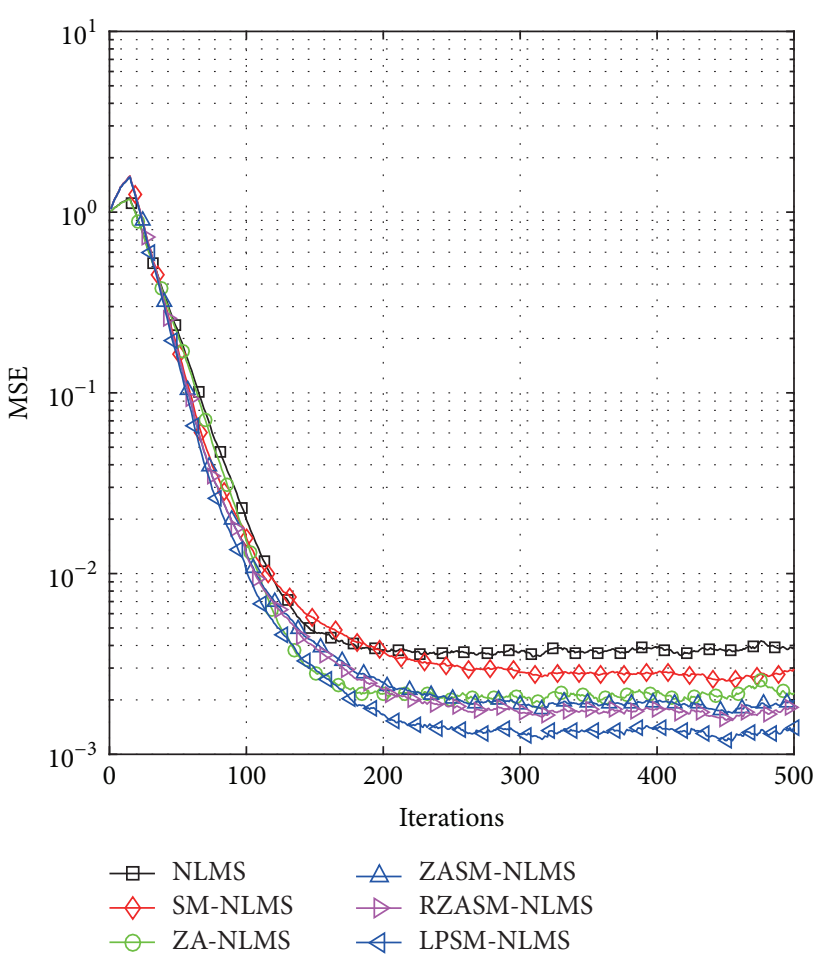

(a) $K=1$

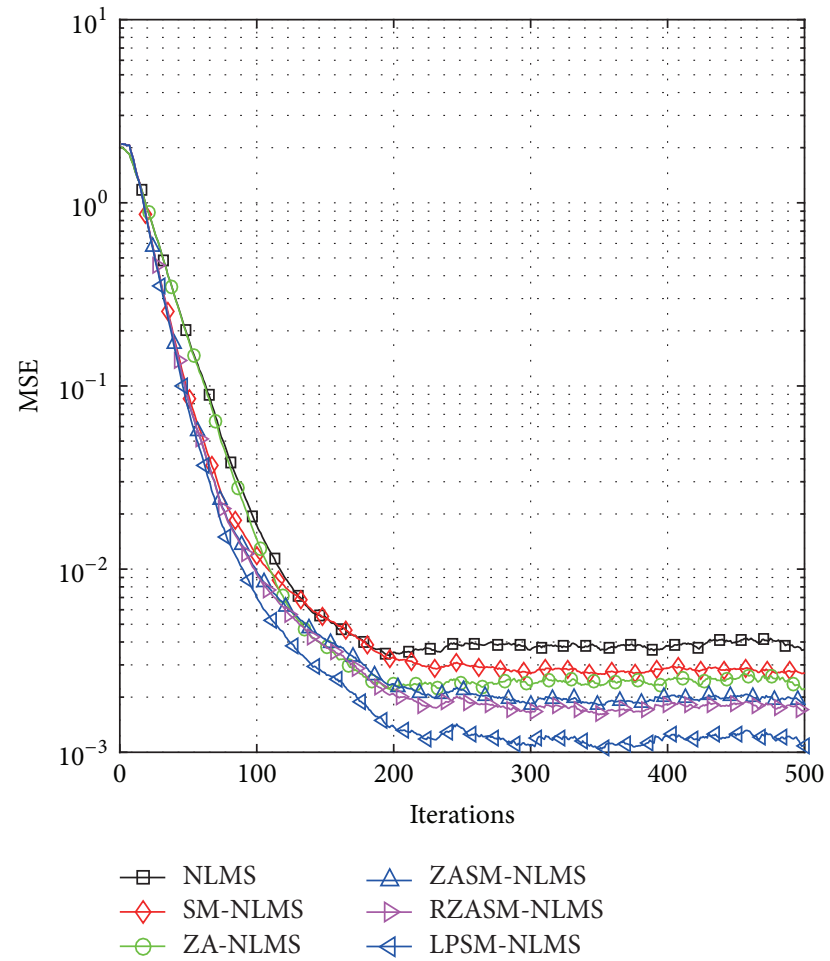

(b) $K=2$

FIGURE 2: Sparse channel estimation behavior for SNR $=20 \mathrm{~dB}$. 


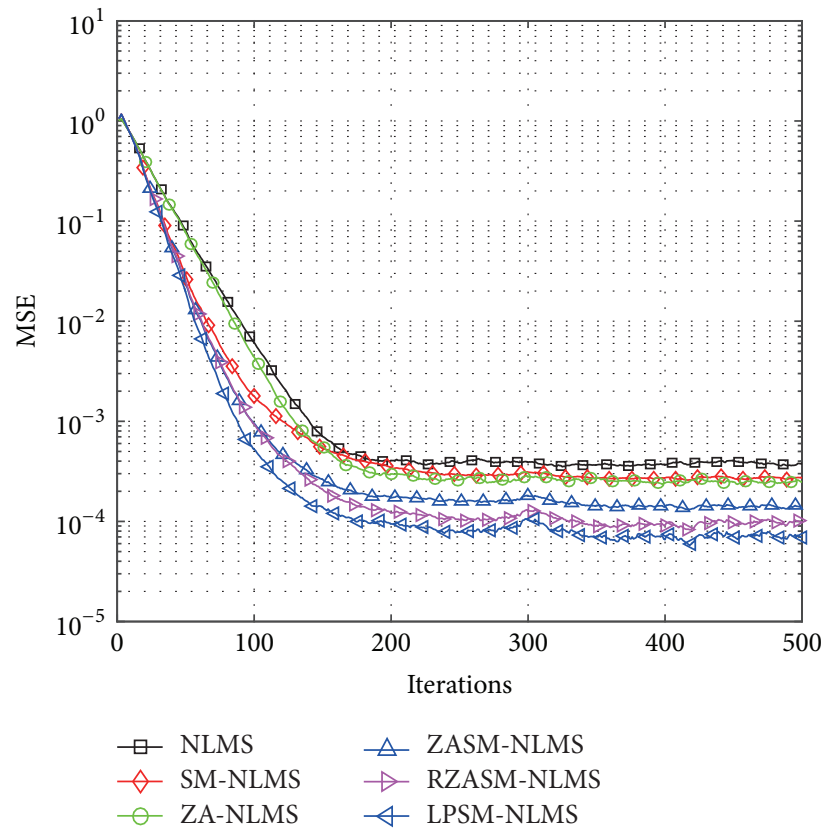

(a) $K=1$

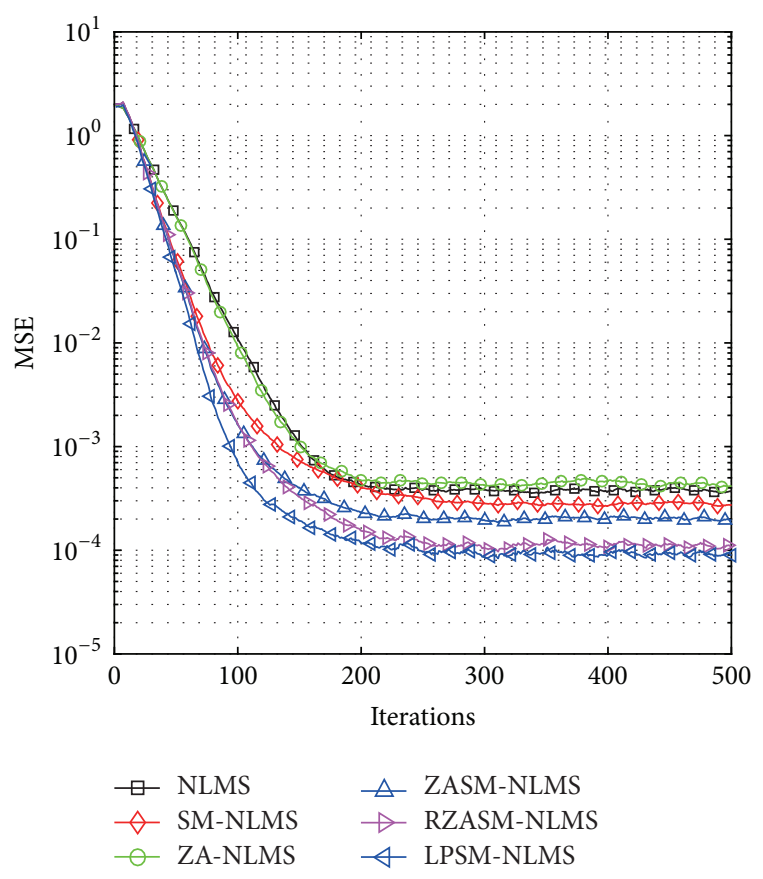

(b) $K=2$

FIGURE 3: Sparse channel estimation behavior for $\mathrm{SNR}=30 \mathrm{~dB}$.

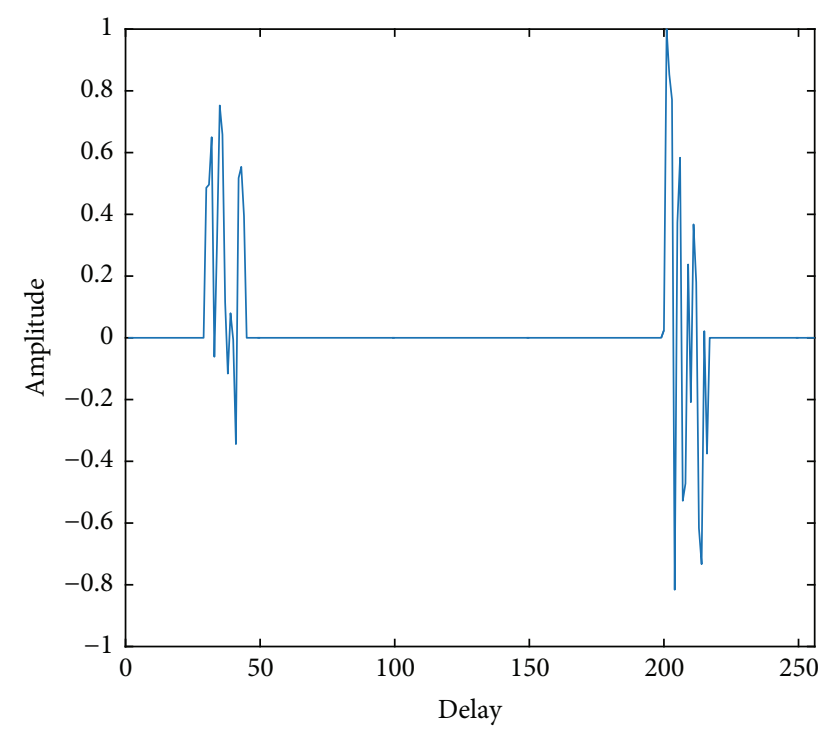

FIGURE 4: A typical network echo channel.

algorithm. Based on the performance of channel estimations obtained from the simulation results, we can say that our proposed LPSM-NLMS algorithm has less effect on the sparseness measure and is robust for sparse channel estimation applications.

\section{Conclusion}

In this paper, a robust LPSM-NLMS algorithm has been proposed and its channel estimation behaviors have been

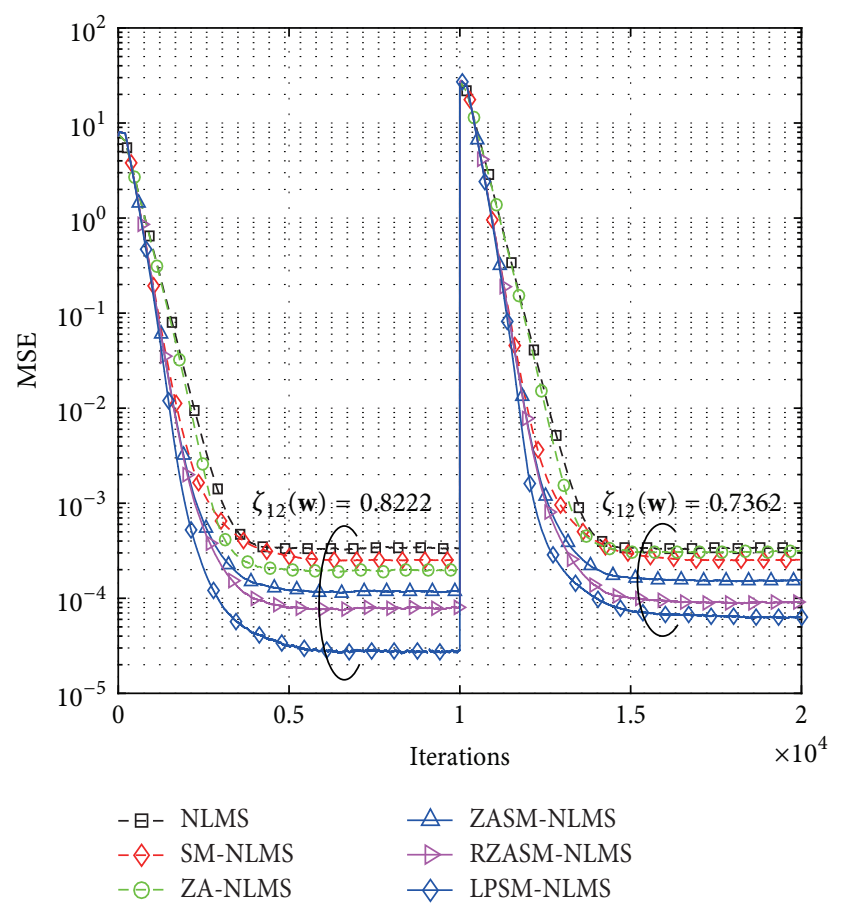

FIGURE 5: Tracking performance of a 256-tap network echo path driven by white input.

evaluated over a sparse multipath channel, a typical network echo channel, and a room echo channel. A $l_{p}$-norm constraint of the channel coefficients has been utilized and incorporated into the cost function of the traditional SM-NLMS algorithm 


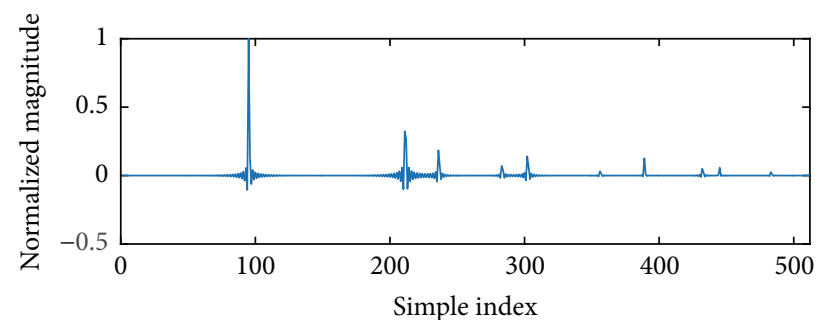

(a) An example of AIR channel

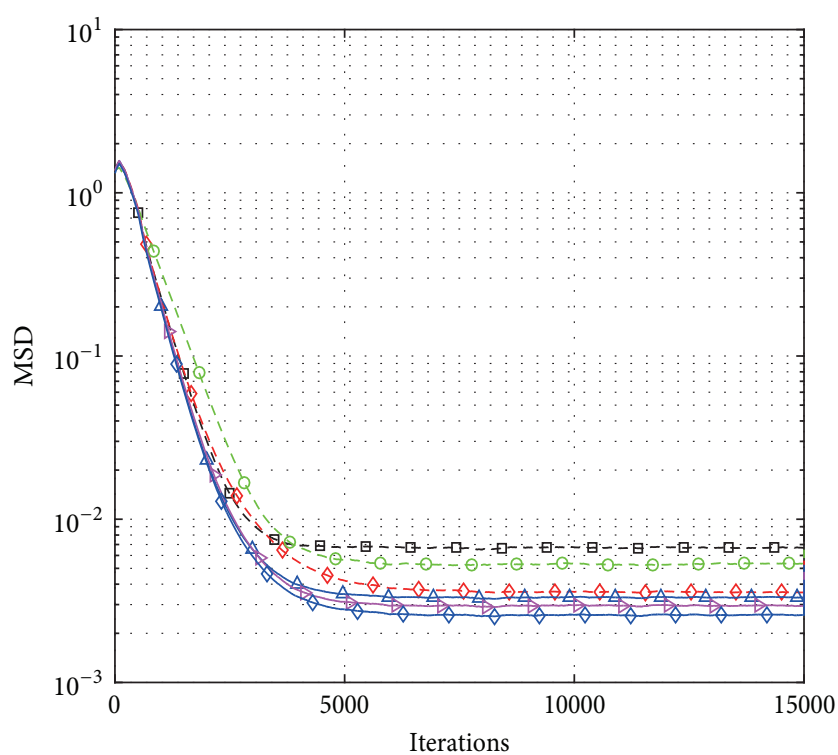

$$
\begin{array}{ll}
-\bullet-\text { NLMS } & \searrow \text { ZASM-NLMS } \\
-\diamond-\text { SM-NLMS } & \triangleright \text { RZASM-NLMS } \\
-\ominus-\text { ZA-NLMS } & \diamond \text { LPSM-NLMS }
\end{array}
$$

(b) MSE of the proposed LPSM-NLMS algorithm

FIGURE 6: Performance of the proposed LPSM-NLMS algorithm for estimating an AIR channel.

to construct the proposed LPSM-NLMS algorithm. As a result, a flexible zero attractor was given in the iteration. The computer simulation results help to verify the fact that the proposed LPSM-NLMS algorithm has better convergence speed and channel estimation behavior in comparison with the previously proposed sparse SM-NLMS and traditional SM-NLMS and NLMS algorithms.

\section{Competing Interests}

The authors declare that there is no conflict of interests regarding the publication of this article.

\section{Acknowledgments}

This work was partially supported by the Navy Defense Foundation of China (4010403020102 and 4010403020103), National Natural Science Foundation of China (61571149), the Science and Technology innovative Talents Foundation of Harbin (2013RFXXJ083 and 2016RAXXJ044), International Science and Technology Cooperation Program of China (2014DFR10240), Projects for the Selected Returned Overseas Chinese Scholars of Heilongjiang Province of China, and the Foundational Research Funds for the Central Universities (HEUCF131602 and HEUCFD1433).

\section{References}

[1] S. Gollamudi, S. Nagaraj, S. Kapoor, and Y.-F. Huang, "Setmembership filtering and a set-membership normalized LMS algorithm with an adaptive step size," IEEE Signal Processing Letters, vol. 5, no. 5, pp. 111-114, 1998.
[2] J. R. Deller, "Set membership identification in digital signal processing," IEEE ASSP Magazine, vol. 6, no. 4, pp. 4-20, 1989.

[3] P. S. R. Diniz, Adaptive Filtering: Algorithms and Practical Implementation, Springer, 4th edition, 2013.

[4] S. Zhang and J. Zhang, "Set-membership NLMS algorithm with robust error bound," IEEE Transactions on Circuits and Systems II: Express Briefs, vol. 61, no. 7, pp. 536-540, 2014.

[5] Md. Z. A. Bhooto and A. Antoniou, "A set-membership NLMS algorithm with adaptive error bound," in Proceedings of the Canadian Conference on Electrical and Computer Engineering (CCECE '08), Niagara Falls, Canada, May 2008.

[6] R. C. de Lamare and P. S. R. Diniz, "Set-membership adaptive algorithms based on time-varying error bounds and their application to interference suppression," in Proceedings of the International Telecommunications Symposium (ITS '06), pp. 884-888, Fortaleza, Brazil, September 2006.

[7] G. Gui and F. Adachi, "Improved least mean square algorithm with application to adaptive sparse channel estimation," Eurasip Journal on Wireless Communications and Networking, vol. 2013, no. 1, article 204, 2013.

[8] J. G. Proakis, Digital Communications, McGraw-Hill, 4th edition, 2001.

[9] F. Adachi and E. Kudoh, "New direction of broadband wireless technology," Wireless Communications and Mobile Computing, vol. 7, no. 8, pp. 969-983, 2007.

[10] L. Vuokko, V.-M. Kolmonen, J. Salo, and P. Vainikainen, "Measurement of large-scale cluster power characteristics for geometric channel models," IEEE Transactions on Antennas and Propagation, vol. 55, no. 11, pp. 3361-3365, 2007.

[11] S. F. Cotter and B. D. Rao, "Sparse channel estimation via matching pursuit with application to equalization," IEEE Transactions on Communications, vol. 50, no. 3, pp. 374-377, 2002. 
[12] P. Maechler, P. Greisen, B. Sporrer, S. Steiner, N. Felber, and A. Burg, "Implementation of greedy algorithms for LTE sparse channel estimation," in Proceedings of the 44th Asilomar Conference on Signals, Systems and Computers (Asilomar '10), pp. 400-405, Pacific Grove, Calif, USA, November 2010.

[13] J. Meng, W. Yin, Y. Li, N. T. Nguyen, and Z. Han, “Compressive sensing based high-resolution channel estimation for OFDM system," IEEE Journal of Selected Topics in Signal Processing, vol. 6, no. 1, pp. 15-25, 2012.

[14] W. U. Bajwa, J. Haupt, A. M. Sayeed, and R. Nowak, "Compressed channel sensing: a new approach to estimating sparse multipath channels," Proceedings of the IEEE, vol. 98, no. 6, pp. 1058-1076, 2010.

[15] G. Tauböck and F. Hlawatsch, "A compressed sensing technique for OFDM channel estimation in mobile environments: exploiting channel sparsity for reducing pilots," in Proceedings of the 2008 IEEE International Conference on Acoustics, Speech and Signal Processing (ICASSP '08), pp. 2885-2888, Las Vegas, Nev, USA, April 2008.

[16] Y. Chen, Y. Gu, and A. O. Hero, "Sparse LMS for system identification," in Proceedings of the IEEE International Conference on Acoustics, Speech, and Signal Processing (ICASSP '09), pp. 31253128, Taipei, Taiwan, April 2009.

[17] K. Shi and P. Shi, "Convergence analysis of sparse LMS algorithms with $l_{1}$-norm penalty based on white input signal," Signal Processing, vol. 90, no. 12, pp. 3289-3293, 2010.

[18] O. Taheri and S. A. Vorobyov, "Sparse channel estimation with $L p$-norm and reweighted $L 1$-norm penalized least mean squares," in Proceedings of the IEEE International Conference on Acoustic Speech and Signal Processing (ICASSP '11), pp. 28642867, Prague, Czech Republic, May 2011.

[19] Y. Gu, J. Jin, and S. Mei, " $l_{0}$ norm constraint LMS algorithm for sparse system identification," IEEE Signal Processing Letters, vol. 16, no. 9, pp. 774-777, 2009.

[20] R. Niazadeh, S. H. Ghalehjegh, M. Babaie-Zadeh, and C. Jutten, "ISI sparse channel estimation based on SL0 and its application in ML sequence-by-sequence equalization," Signal Processing, vol. 92, no. 8, pp. 1875-1885, 2012.

[21] Y. Li and M. Hamamura, "Zero-attracting variable-step-size least mean square algorithms for adaptive sparse channel estimation," International Journal of Adaptive Control and Signal Processing, vol. 29, no. 9, pp. 1189-1206, 2015.

[22] Y. Li, Y. Wang, and T. Jiang, "Norm-adaption penalized least mean square/fourth algorithm for sparse channel estimation," Signal Processing, vol. 128, pp. 243-251, 2016.

[23] Y. Li, Y. Wang, and T. Jiang, "Sparse-aware set-membership NLMS algorithms and their application for sparse channel estimation and echo cancelation," AEU-International Journal of Electronics and Communications, vol. 70, no. 7, pp. 895-902, 2016.

[24] E. J. Candès, "The restricted isometry property and its implications for compressed sensing," Comptes Rendus Mathematique, vol. 346, no. 9-10, pp. 589-592, 2008.

[25] D. L. Duttweiler, "Proportionate normalized least-meansquares adaptation in echo cancelers," IEEE Transactions on Speech and Audio Processing, vol. 8, no. 5, pp. 508-517, 2000.

[26] Y. Li and M. Hamamura, "An improved proportionate normalized least-mean-square algorithm for broadband multipath channel estimation," The Scientific World Journal, vol. 2014, Article ID 572969, 9 pages, 2014.
[27] R. Meng, R. C. De Lamare, and V. H. Nascimento, "Sparsityaware affine projection adaptive algorithms for system identification," in Proceedings of the Sensor Signal Processing for Defence (SSPD '11), pp. 1-5, London, UK, September 2011.

[28] Y. Li, C. Zhang, and S. Wang, "Low-complexity non-uniform penalized affine projection algorithm for sparse system identification," Circuits, Systems, and Signal Processing, vol. 35, no. 5, pp. 1611-1624, 2016.

[29] M. V. S. Lima, W. A. Martins, and P. S. R. Diniz, "Affine projection algorithms for sparse system identification," in Proceedings of the 2013 38th IEEE International Conference on Acoustics, Speech, and Signal Processing (ICASSP '13), pp. 5666-5670, Vancouver, Canada, May 2013.

[30] Y. Li and M. Hamamura, "Smooth approximation $l_{0}$-norm constrained affine projection algorithm and its applications in sparse channel estimation," The Scientific World Journal, vol. 2014, Article ID 937252, 14 pages, 2014.

[31] G. Gui and F. Adachi, "Sparse least mean fourth algorithm for adaptive channel estimation in low signal-to-noise ratio region," International Journal of Communication Systems, vol. 27, no. 11, pp. 3147-3157, 2014.

[32] Y. Li, Y. Wang, and T. Jiang, "Sparse channel estimation based on a p-norm-like constrained least mean fourth algorithm," in Proceedings of the 7th International Conference on Wireless Communications and Signal Processing (WCSP '15), Nanjing, China, October 2015.

[33] G. Gui, A. Mehbodniya, and F. Adachi, "Least mean square/ fourth algorithm for adaptive sparse channel estimation," in Proceedings of the 2013 IEEE 24th Annual International Symposium on Personal, Indoor, and Mobile Radio Communications (PIMRC '13), pp. 296-300, London, UK, September 2013.

[34] Y. Li, Y. Wang, and F. Albu, "Sparse channel estimation based on a reweighted least-mean mixednorm adaptive filter algorithm," in Proceedings of the European Signal Processing Conference (EUSIPCO '16), pp. 2380-2384, Budapest, Hungary, AugustSeptember 2016.

[35] S. Haykin, Adaptive Filter Theory, Prentice Hall, New Jersey, NJ, USA, 1991.

[36] R. Tibshirani, "Regression shrinkage and selection via the lasso," Journal of the Royal Statistical Society. Series B. Methodological, vol. 58, no. 1, pp. 267-288, 1996.

[37] D. L. Donoho, "Compressed sensing," IEEE Transactions on Information Theory, vol. 52, no. 4, pp. 1289-1306, 2006.

[38] M. S. Salman, "Sparse leaky-LMS algorithm for system identification and its convengence analysis," International Journal of Adaptive Control and Signal Processing, vol. 28, no. 10, pp. 10651072, 2014.

[39] P. O. Hoyer, "Non-negative matrix factorization with sparseness constraints," Journal of Machine Learning Research, vol. 5, pp. 1457-1469, 2004.

[40] J. B. Allen and D. A. Berkley, "Image method for efficiently simulating small-room acoustics," The Journal of the Acoustical Society of America, vol. 65, no. 4, pp. 943-950, 1979.

[41] P. Loganathan, E. A. P. Habets, and P. A. Naylor, "A proportionate adaptive algorithm with variable partitioned block length for acoustic echo cancellation," in Proceedings of the 36th IEEE International Conference on Acoustics, Speech, and Signal Processing (ICASSP '11), pp. 73-76, Prague, Czech Republic, May 2011. 


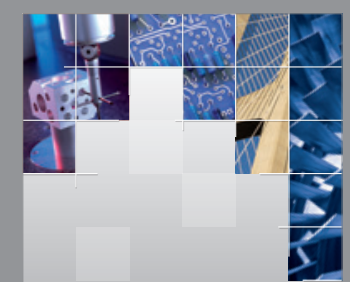

\section{Enfincering}
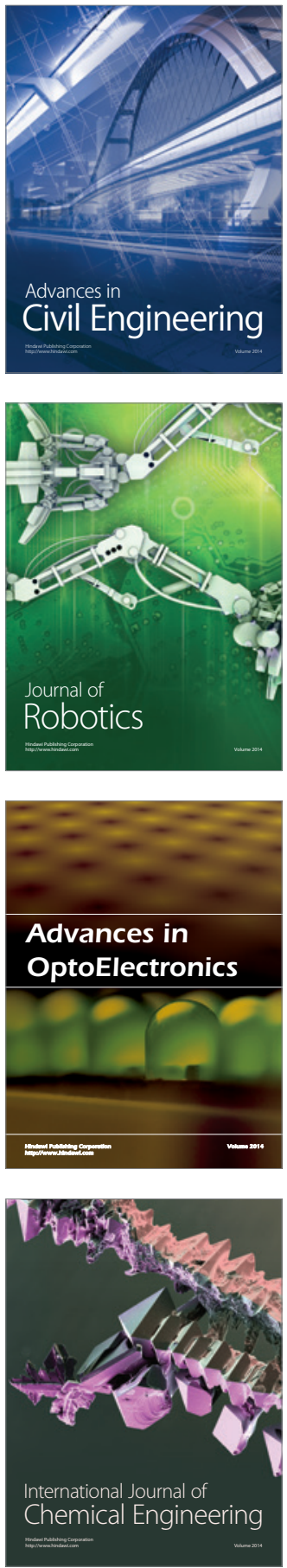

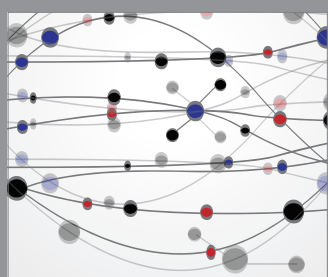

The Scientific World Journal

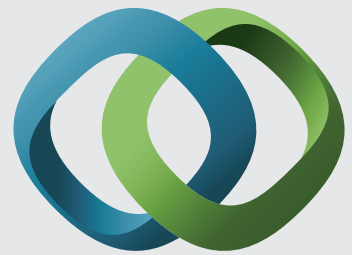

\section{Hindawi}

Submit your manuscripts at

https://www.hindawi.com
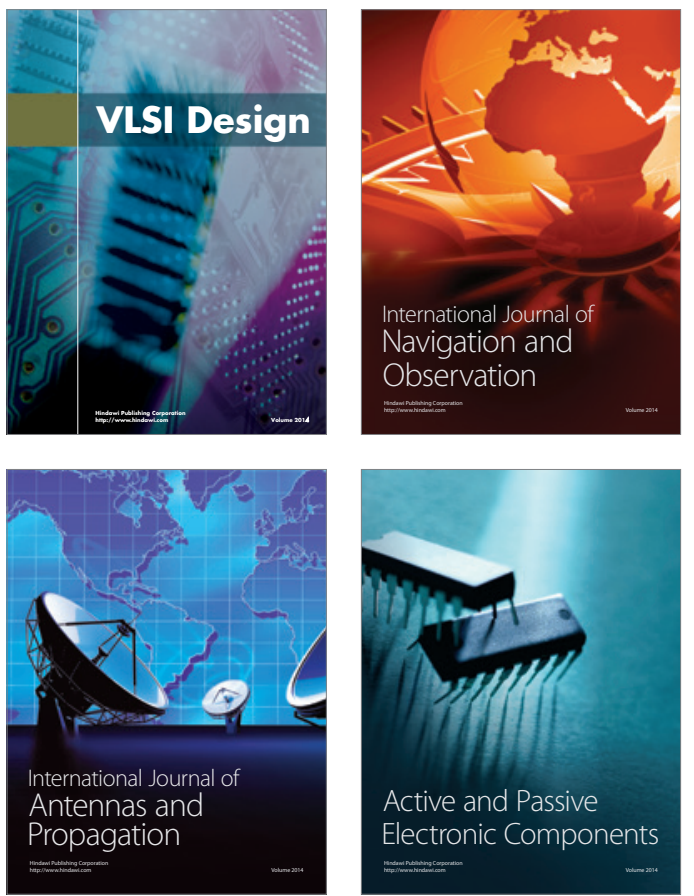
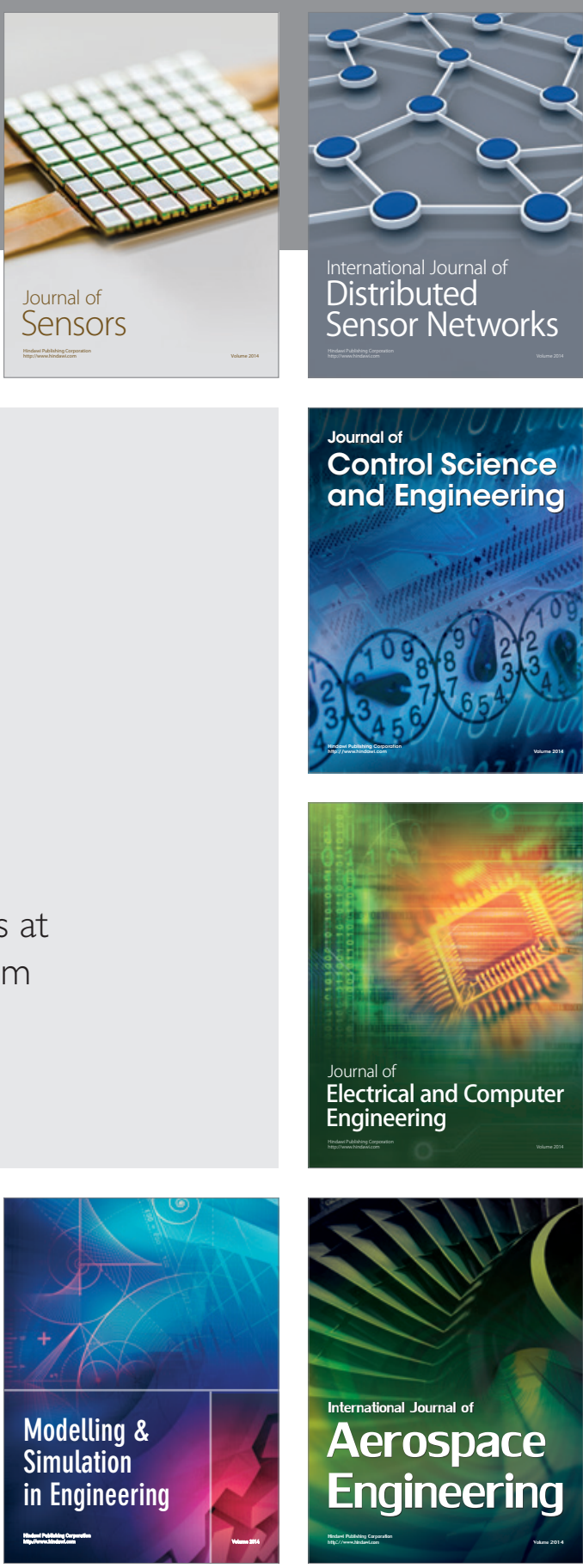

International Journal of

Distributed

Sensor Networks

$-$

Joumal of

Control Science

and Engineering
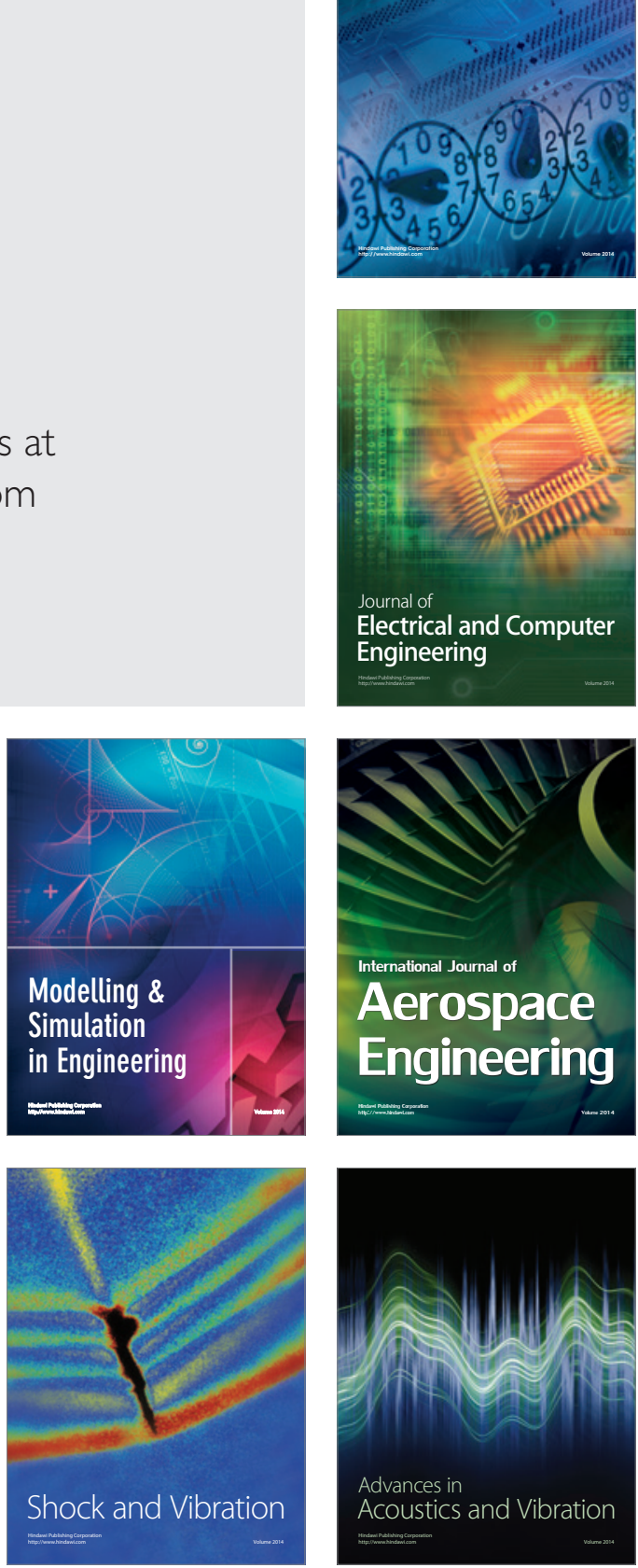